\title{
Identification of Dermatophytes Isolated from Tinea Capitis Patients and Their in vitro Susceptibility to Terbinafine
}

\author{
Khaled M. Tawfik ${ }^{1}$ MD, Refaat R. Mohammed ${ }^{1}$ MD, Asmaa S. Shaltout ${ }^{2}$ MD.
}

\author{
* Corresponding Author: \\ Asmaa S. Shaltout \\ asmaashaltout@aun.edu.eg \\ Received for publication March \\ 12, 2021; Accepted June 5, 2021; \\ Published online June 5, 2021, \\ 2021.
}

Copyright The Authors
published by Al-Azhar
University, Faculty of Medicine,
Cairo, Egypt. Users have the
right to read, download, copy,
distribute, print, search, or link
to the full texts of articles under
the following conditions:
Creative Commons Attribution-
Share Alike 4.0 International
Public License (CC BY-SA 4.0).
doi: 10.21608/aimj.2021.56585.1435.
${ }^{1}$ Dermatology, Andrology and STDs,
Faculty of Medicine, Al-Azhar
University, Assiut, Egypt.
${ }^{2}$ Medical Microbiology and
Immunology Department
Department, Faculty of Medicine,
Assiut University, Assiut, Egypt

\section{INTRODUCTION}

Tinea capitis is a common dermatophyte infection of the scalp hair and mainly happening at a young age ${ }^{1}$. Its clinical manifestations are determined through hair invasion by the dermatophyte and the host's immune response, ranging from mild scaling with little hair loss to severe inflammatory and pustular presentations with scarring alopecia ${ }^{2}$. Tinea capitis caused by Trichophyton (T), and Microsporum (M) dermatophytes genera can dissolve keratinized tissue 1

Antifungal drugs that are used to treat dermatophytosis direct the ergosterol synthesis pathway. Azoles inhibit lanosterol 14- $\alpha$-demethylase, leading to the aggregation of sterol precursors, resulting in disruption of plasma membrane structure and function ${ }^{3}$. In contrast, terbinafine belongs to
Background: Tinea capitis is an infection caused by dermatophyte fungi of scalp hair follicles and the neighboring skin. Antifungal drugs as the ergosterol Aim of the study: The current study aimed to identify species and in vitro antifungal susceptibility profile of terbinafine on dermatophytes clinical isolates accompanied by sequencing of the highest minimal

Patients and Methods: The present study is a descriptive cross-sectional was conducted at Al-Azhar Hospital outpatient clinic, in the period Souraud's dextrose agar followed by in vitro susceptibility testing to Results: The isolated dermatophyte species were 16 (32\%) isolates of $M$. canis and 23(46\%) isolates of T.violaceum. The MIC varied from 0.003 $.092 \mu \mathrm{g} / \mathrm{ml}$ for M.canis and from $0.005-0.116 \mu \mathrm{g} / \mathrm{ml}$ for T.violaceum. It was found in $M$. canis strain has two base variations with type strain representative isolates with the highest MIC.

. sensitivity to the antifungal agents. By comparing the $M$. canis and $T$. violaceum isolates with type strain on GenBank, some base-pair substitutions were found.

Keywords: tinea capitis; dermatophytes; terbinafine

Disclosure: The authors have no financial interest to declare in relation to the content of this article. The Article Processing Charge was paid for by the authors.

Authorship: All authors have a substantial contribution to the article.

allylamines inhibits squalene epoxidase enzyme, which is involved in the initial steps of ergosterol biosynthesis ${ }^{4}$. This inhibition results in the aggregation of squalene and fungal growth inhibition 5

However, the effect of the drug is different according to the infectious agent. Terbinafine was reported to be higher in treating $T$. tonsurans and $T$. violaceum, while griseofulvin was higher in treating $M$. canis and other Microsporum species ${ }^{6,7}$. Lipozencic et al. ${ }^{8}$ showed that the use of terbinafine for a long duration does not enhance its activity against Microsporum species, concluding that terbinafine not recommended as a first- line in treating tinea capitis caused by Microsporum species

Therefore, the importance of early identification of dermatophyte species and their antifungal susceptibility profiles is mandatory for establishing ways to control the spread of dermatophytosis ${ }^{9}$. 
This study was conducted to identify species and in vitro antifungal susceptibility profile of terbinafine on dermatophytes, accompanied by sequencing of the highest minimal inhibitory concentration (MIC) isolates.

\section{PATIENTS AND METHODS}

A descriptive cross-sectional study was conducted at Al-Azhar Hospital outpatient clinic, Al Azhar University (Assiut), and Assiut University Mycology Center (AUMC), in the period from the 1st of July 2019 to the 15th of July 2020. The study protocol was reviewed and approved by the Ethical Review Committee of $\mathrm{Al}$ Azhar Faculty of Medicine (Assiut). All participants have signed informed consent.

\section{Study Participants}

Fifty patients suspected clinically as tinea capitis infection were asked to participate in the study. Data was taken from the patients, including age, sex, presenting symptoms, and signs. Patients who received antifungal therapies in the previous two weeks were kept out from the study.

\section{Sample Taking and Transport}

The suspected tinea capitis lesions were cleaned with $70 \%$ ethyl alcohol, then hair, including the roots, was plucked for hair samples, a sterile scalpel was used to collect scales from the scalp. Samples were transported in sterile plastic petri dishes and were divided between microscopic examination and culture.

\section{Microscopic Examination and Culture of Collected Samples}

Direct microscopic examination was done to all samples using $20 \%$ potassium hydroxide $(\mathrm{KOH})$ and lactophenol cotton blue (LCB) followed by gentle heating for 3-4 seconds. Every sample was inoculated on Sabouraud's dextrose agar (SDA) plates (Himedia Company, India). Chloramphenicol $(0.5 \mathrm{gm} / \mathrm{L})$ was added to prevent bacterial contamination and to prevent saprophytic fungi cycloheximide $(0.5 \mathrm{gm} / \mathrm{L})$ was added. Incubation of plates at $28^{\circ} \mathrm{C}$ for at least six weeks was done ${ }^{10}$.

\section{In vitro Susceptibility Testing to terbinafine}

Antifungal activity study was performed in vitro against six isolates of Microsporum canis and six of T. violaceum using modified agar cup diffusion method. The isolates were subcultured on SDA at $28^{\circ} \mathrm{C}$, and dermatophyte suspension was prepared in Sabouraud's broth by smoothly scraping the colonies' surface with a sterile cotton swab then vortex and permitted to settle. The final inoculum concentration was adjusted to $1 \times 10^{3}$ to $3 \times 10^{3} \mathrm{CFU} / \mathrm{mL}$. SDA was poured into Petri dishes at $50-70{ }^{\circ} \mathrm{C}$, and it was left to solidify under ultraviolet rays in sterile category II laminar flow for 15 min. Standardized inoculum 300ul was streaked by cotton swab over the agar medium. Cylindrical plugs were removed from the agar by a sterile cork borer. Terbinafine was obtained as a powder (Sigma-Aldrich), a stock was made by dilution with Dimethyl sulfoxide (DMSO) kept at $-70{ }^{\circ} \mathrm{C}$. 50ul of final concentrations in distilled water ranged from $4 \mathrm{ug} / \mathrm{ml}$ to $0.001 \mathrm{ug} / \mathrm{ml}$ were added to the wells. The plates were incubated at $28^{\circ} \mathrm{C}$, and then the inhibition zones were measured in millimeters ${ }^{11}$.

\section{Molecular Identification}

DNA extraction: following the manufacturer's instructions, DNA was extracted by QIAamp DNA Mini Kit extraction kit (QIAGEN, Germany).

\section{Sequencing}

The extracted product was sent to SolGent lab (Daejeon, South Korea). Before sequencing, using two universal fungal primers, Internal transcribed spacer (ITS1) (forward) and ITS4 (reverse), the ribosomal RNA (rRNA) gene was amplified by PCR reaction. Primers used have the following composition: ITS1 (5' - TCC GTA GGT GAA CCT GCG G - 3'), and ITS4 (5'- TCC TCC GCT TAT TGA TAT GC -3') ${ }^{12}$. The PCR reaction was prepared using Solgent EF-Taq (SolGent, Daejeon, South Korea).

\section{RESULTS}

The current work was a descriptive cross-sectional study of 50 patients having clinically suspected tinea capitis infections. Out of these 50 patients, 21 were diagnosed with tinea capitis scaly type while 15 patients were having tinea capitis black dot type with scales. Black dot without scales was noted in 10 patients, and four patients were having kerion.

Data analysis showed that 43 were males (86\%) and 7 were female (14\%). The youngest was 1.4 years old, and the eldest was 14 years old male patients with mean age $6.1 \pm 2.67$ years old. Tinea capitis infections were found to be more prevalent in males than in females.

Out of 50 samples, fungi were demonstrated in $68 \%$ of cases by direct microscopy and $78 \%$ by culture. Both of them were positive in $48 \%$ of the samples. Dermatophyte species isolated from tinea capitis infection were $16(32 \%)$ isolates of $M$. canis and 23(46\%) isolates of T.violaceum, as shown in (Table $1)$.

\begin{tabular}{|l|l|l|}
\hline & $\begin{array}{l}\text { Number and (\%) } \\
\text { of tested patients }\end{array}$ & $\begin{array}{l}\text { Number and (\%) } \\
\text { of Positive cases }\end{array}$ \\
\hline $\begin{array}{l}\text { KOH } \\
\text { smear }\end{array}$ & $50(100 \%)$ & $34(68 \%)$ \\
\hline Culture & $50(100 \%)$ & $\begin{array}{l}23(46 \%) \\
\text { T.violaceum } \\
16(32 \%) \text { M. canis }\end{array}$ \\
\hline
\end{tabular}

Table 1: Showing the number and (\%) of tested and positive patients with fungal growth $(\mathrm{KOH}$ : 
potassium hydroxide, T:Trichophyton, M:Microsporium).

On examination, it was observed that scales and +ve hair pulling were the most common signs in tinea capitis. Also, $100 \%$ of kerion cases were presented with a secondary bacterial infection. Most of the pa- tients were presented with a single lesion ( $\mathrm{n}=32$, $64 \%$ ) whereas multiple lesions were noted in $36 \%$. The type of the lesions was mainly black dot in $50 \%$ $(n=25)$; however, scales were common in both types as shown in (Table 2).

\begin{tabular}{|c|c|c|c|c|c|c|c|}
\hline $\begin{array}{l}\text { Types } \\
\text { Total }=50\end{array}$ & $\begin{array}{l}\text { Scales } \\
\text { only } \\
\text { N (\%) }\end{array}$ & $\begin{array}{l}\text { +ve hair } \\
\text { pulling } \\
\text { only } \\
\mathrm{N}(\%) \\
\end{array}$ & $\begin{array}{l}\text { Both signs } \\
\text { N (\%) }\end{array}$ & $\begin{array}{l}\text { 2ry } \\
\text { infection } \\
\mathrm{N}(\%)\end{array}$ & $\begin{array}{l}\text { 2ry } \\
\text { Parasitic } \\
\text { N (\%) }\end{array}$ & $\begin{array}{l}\text { Itching } \\
\mathrm{N}(\%)\end{array}$ & $\begin{array}{l}\text { Number of } \\
\text { lesions per site }\end{array}$ \\
\hline $\begin{array}{l}\text { Tinea capitis } \\
\text { (scaly type) } \\
\mathrm{N}=21\end{array}$ & 7(33.3\%) & negative & 14(66.7)\% & negative & negative & $2(9.5 \%)$ & $\begin{array}{ll}\text { Single } & 14 \\
(66.7 \%) & \\
\text { Multiple } & \\
7(33.3 \%) & \\
\end{array}$ \\
\hline $\begin{array}{l}\text { Tinea capitis (black } \\
\text { dot type) } \\
\mathrm{N}=25\end{array}$ & negative & $9(36 \%)$ & $15(60 \%)$ & negative & $1(4 \%)$ & $7(28 \%)$ & $\begin{array}{l}\text { Single } 14(56 \%) \\
\text { Multiple } 11(44 \%)\end{array}$ \\
\hline $\begin{array}{l}\text { Kerion } \\
\mathrm{N}=4\end{array}$ & $2(50 \%)$ & $4(100 \%)$ & negative & $4(100 \%)$ & negative & $1(25 \%)$ & Single $4(100 \%)$ \\
\hline
\end{tabular}

Table 2: Showing clinical profile in different clinical presentations $(\mathrm{N}=$ number, $2 \mathrm{ry}=$ secondary)

\section{Identification of Isolated Dermatophyte Species}

Identification of dermatophyte species from clinically suspected tinea capitis was dependent on direct microscopic examination from sample followed by inoculation of collected samples on culture media as shown in (figure 1, 2).

In vitro antifungal susceptibility test showed that al tested isolates were sensitive to terbinafine but at different concentrations. The MIC varied from 0.003 $.092 \mu \mathrm{g} / \mathrm{ml}$ for M.canis and from $0.005-0.116 \mu \mathrm{g} / \mathrm{ml}$ for T.violaceum as shown in (Table 3).
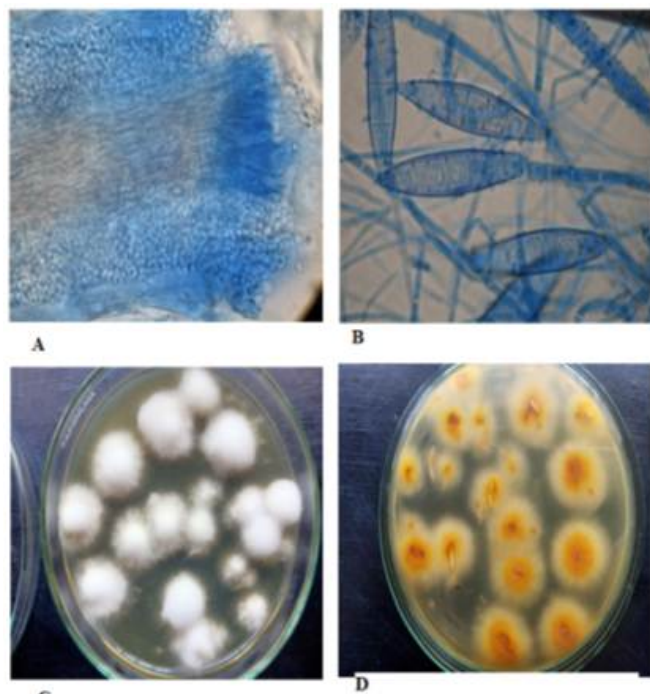

Fig 1: Microscopic and macroscopic appearance of Microsporum canis. (A) Ectothrix hair invasion pattern (B) Numerous spindle- shaped macroconidia, ,thick-walled (C) White fluffy, a furlike colony with feathery border (D) reverse deep yellow.
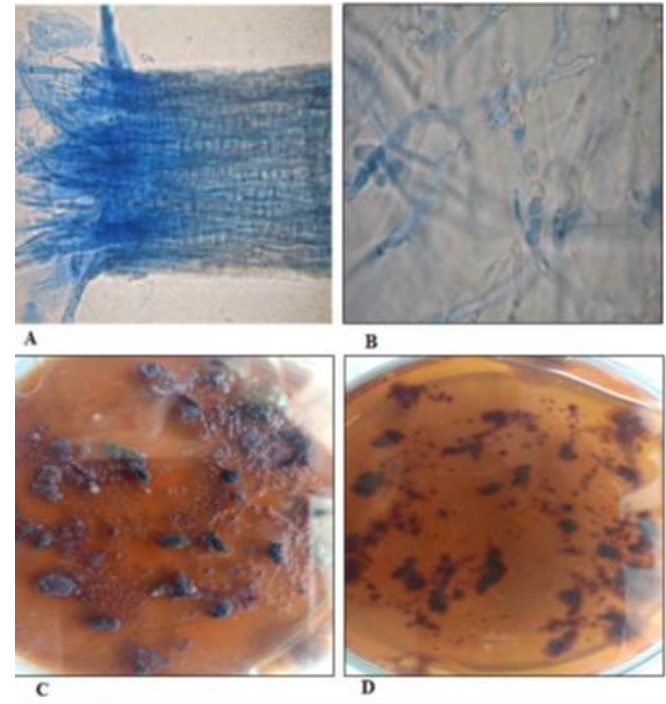

B

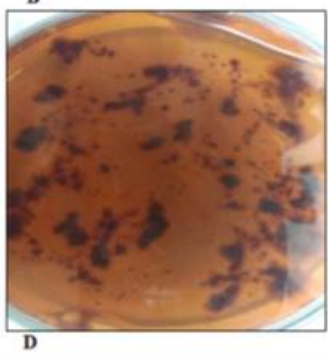

Fig 2: Microscopic and macroscopic appearance of Trichophyton violaceum. (A) Endothrix hair invasion pattern (B) Irregular hyphae with intercalary chlamydospores. (C)Waxy, cerebriform, heaped purple colony; (D) reverse deep port wine.

\section{DNA sequencing}

This was done only for two representative isolates with the highest MIC results. M. canis was identical by $99.28 \%$ with the type strain (NR_131265). $T$. violaceum was identical by $98.82 \%$ with the type material of $T$. violaceum (NR_144901). The query sequences were compared with those in the GenBank database by CLUSTALW analysis. It was found in $M$. canis strain two bases variations at positions 679 , 680 and extra-base at position 678 in the alignment of ITS sequences of with type strain (NR_131265) from GenBank. Whereas T.violaceum strain has 10 base variations at positions 648, 645, 643, 642, 640, 
638, 637, 636, 633, 631 in comparison with type strain (NR_144901).

\begin{tabular}{|l|l|}
\hline Number of isolates (12) & MIC ug/ml \\
\hline M.canis & 0.092 \\
\hline M.canis & 0.026 \\
\hline M.canis & 0.026 \\
\hline M.canis & 0.003 \\
\hline M.canis & 0.014 \\
\hline M.canis & 0.042 \\
\hline T.violaceum & 0.025 \\
\hline T.violaceum & 0.005 \\
\hline T.violaceum & 0.022 \\
\hline T.violaceum & 0.058 \\
\hline T.violaceum & 0.032 \\
\hline T.violaceum & 0.116 \\
\hline
\end{tabular}

Table 3: Results of the Minimal Inhibitory Concentration of terbinafine antifungal in 12 isolates from tinea capitis patients. (MIC: Minimal Inhibitory Concentration, M:Microsporum, T:Trichophyton)

\section{DISCUSSION}

In developing countries, tinea capitis is a public health problem ${ }^{13}$. In the present study high occurrence of tinea capitis was in males than females. Bassyouni et al. ${ }^{14}$ reported that males to females ratio was 5:1. They attributed their result to that girls apply vegetable oils on the scalp, described to have some antifungal effects against dermatophytes ${ }^{15,16}$. This study showed that tinea capitis predominate in children. This is in agreement with the previous study, showed that occurance of tinea capitis mainly in children less than ten years of age ${ }^{17}$. Despite the most common clinical type a black dot, scales were the main clinical presentation. This, following the study of El-Khalawany et al. ${ }^{18}$ who reported that scaly scalp was the most clinical presentation in Egypt.

In this study, the commonest isolated fungal pathogen was $T$. violaceum (46\%) and after that M.canis (32\%), this was consistent with the previous multicenter study was done in Egypt by ElKhalawany et al. ${ }^{18}$. T. violaceum was also reported as the most common species in several countries. In Ethiopia, T. violaceum was detected as the most common fungal species $(81.6 \%)^{19}$. Gargoom et al. ${ }^{20}$ also reported that $T$. violaceum is the most common pathogen of Tinea capitis in Libya (50\%). Despite in Kuwait, T. violaceum was not commonly isolated $(19.3 \%)^{21}$.

Conversely, a study was done in some Egyptian schools by Bassyouni et al. ${ }^{14}$ found that M.canis was the most common isolated species. It may be due to contact with animals and sharing towels and caps among pupils. Positive cultures were obtained only in $78 \%$ of samples, which could be explained by applying homemade products before seeking medical advice.

The MIC results with terbinafine to $M$. canis were $0.003-.092 \mathrm{ug} / \mathrm{ml}$. This is in agreement with the data published by Butty et al. ${ }^{22}$ who found that MIC to $M$. canis was $0.01-0.08 \mathrm{ug} / \mathrm{ml}$. Conversely, Mock et al. ${ }^{23}$ reported higher MIC results to M. canis 0.05$0.5 \mathrm{ug} / \mathrm{ml}$. T.violaceum MIC was 0.005-0.116 ug/ml. This consistent with the results of Badali et al. ${ }^{24}$, who reported that MIC results to T.violaceum were $0.016-0.125 \mathrm{ug} / \mathrm{ml}$.

The MIC results of terbinafine showed sensitivity to the antifungal. Terbinafine has been reported to be an effective antifungal agent in treating several dermatophytoses due to Trichophyton ${ }^{24,25}$. However, Yamada et al. ${ }^{26}$ found that specific point mutations in squalene epoxidase genes were the cause of terbinafine resistance in Trichophyton clinical isolates. Insensitivity to terbinafine after treatment for 1.5-3 months in tinea capitis patients caused by $M$. canis has been reported ${ }^{27}$. Kano et al., ${ }^{28}$ explained that by overexpression of genes encoding $\mathrm{ABC}$ transporter family in patients received long terbinafine treatment duration.

By comparing the $M$. canis and T. violaceum isolates with type strain on GenBank, some base-pair substitutions were found. Neither the phenotypic nor the molecular identification was affected by these substitutions. Also, it may be related to relatively high MIC detected in sequenced trichophyton strain. Osborne et al. ${ }^{29}$ reported the silent nucleotide substitution of about 20 times common than nonsilent nucleotide substitution rates. Singh et al. ${ }^{30}$ estimated that the terbinafine resistance mechanism has been assigned to point mutations in the squalene epoxidase gene that leads to single amino acid substitutions. Squalene epoxidase is a key enzyme in the ergosterol synthesis pathway. Further sequencing to the squalene epoxidase gene will be required to fully elucidate this point.

Despite sequencing providing a highly accurate method for dermatophytes' characterization, it is very expensive to be implemented in routine identification. Therefore, it is suggested when atypical dermatophytes are isolated ${ }^{31}$.

\section{CONCLUSION}

This study highlights the isolation of certain species, such as $M$. canis and $T$. violaceum. T. violaceum is prevalent in this region. Terbinafine exhibited good in vitro activity to $M$. canis and $T$. violaceum species.

\section{REFERENCES}

1. Elewski BE. Tinea capitis: a current perspective. J Am Acad Dermatol. 2000; 42 (1):1-20. 
2. Farooqi M, Tabassum S, Rizvi DA, et al. Clinical types of tinea capitis and species identification in children: an experience from tertiary care centres of Karachi, Pakistan. J Pak Med Assoc. 2014; 64 (3):304-8.

3. Kathiravan MK, Salake AB, Chothe AS, et al The biology and chemistry of antifungal agents: a review. Bioorg. Med. Chem. 2012; 20 (19):567898.

4. Odds FC, Brown AJ and Gow NA. Antifungal agents: mechanisms of action. Trends Microbiol. 2003; 11 (6):272-9.

5. Takami T, Fang Y, Zhou X, et al. A genetic and pharmacological analysis of isoprenoid pathway by LC-MS/MS in fission yeast. PLoS One. 2012; 7 (11):49004.

6. Gupta AK and Drummond analysis of randomized, controlled trials comparing particular doses of griseofulvin and terbinafine for the treatment of tinea capitis. Pediatr. Dermatol. 2013; 30(1):1-6.

7. Chen $X$, Jiang $X$, Yang $M$, et al. Systemic antifungal therapy for tinea capitis in children: An abridged Cochrane Review. J Am Acad Dermatol. 2017; 76 (2):368-74.

8. Lipozencic J, Skerlev M, Orofino Tinea Capitis Study Group. A randomized, double

study of oral terbinafine and open

dose griseofulvin in children with tinea capitis due to Microsporum species. Br. J. Dermatol. 2002; 146 (5):816-23.

9. Sharifzadeh A, Shokri H and Khosravi AR. In vitro evaluation of antifungal susceptibility and keratinase, elastase, lipase and DN ase activities of different dermatophyte species isolated from clinical specimens in Iran. Mycoses. 2016; 59 (11):710-9.

10. Abdel-Sater MA, Moubasher AA and Soliman Z. Identification of three yeast species using the conventional and internal transcribed spacer region sequencing methods as first or second global record from human superficial infections. Mycoses. 2016; 59 (10):652-61.

11. Ginovyan M, Keryan A, Bazukyan I, et al. The large scale antibacterial, antifungal and antiphage efficiency of Petamcin-A: new multicomponent preparation for skin diseases treatment. Ann Clin Microbiol Antimicrob. 2015;14 (1):1-7.

12. Shehata AS, Mukherjee PK, Aboulatta HN, et al Single-step PCR using (GACA) 4 primer: utility for rapid identification of dermatophyte species and strains. J. Clin. Microbiol. 2008; 46 (8):2641-5.

13. Kechia FA, Kouoto EA, Nkoa T, Nweze EI, Fokoua DC, et al. Epidemiology of tinea capitis among school-age children in Meiganga, Cameroon. J Mycol Med. 2014; 24 (2):129-34.

14. Bassyouni RH, El-Sherbiny NA, Abd El Raheem TA, et al. Changing in the epidemiology of tinea capitis among school children in Egypt. Ann Dermatol. 2017; 29 (1):13.

15. Vijayakumar R, Muthukumar C, Kumar T, et al. Characterization of Malassezia furfur and its control by using plant extracts. Indian $J$. Dermatol. 2006; 51 (2):145.
16. Geweely NS. Antifungal activity of ozonized olive oil (Oleozone). Int J Agri Biol. 2006; 8 (5):671-8.

17. Sidat MM, Correia D and Buene TP. Tinea capitis among children at one suburban primary school in the city of Maputo, Mozambique. Rev Soc Bras Med Trop. 2007; 40 (4):473-5.

18. El-Khalawany M, Shaaban D, Hassan H, et al. A multicenter clinicomycological study evaluating the spectrum of adult tinea capitis in Egypt. Acta Dermatovenerol Alp Pannonica Adriat. 2013; 22 (4):77-82.

19. Woldeamanuel Y, Mengistu Y, Chryssanthou E, et al. Dermatophytosis in Tulugudu Island, Ethiopia. Med Mycol. 2005;43:79-82.

20. Gargoom AM, Elyazachi MB, Al-Ani SM, et al. - Mair Tinea capitis in Benghazi, Libya. Int J Dermatol. 2000; 39:263-5.

21. Adel A, Sultan A, Basmiah A, et al. Prevalence of tinea capitis in southern Kuwait. Mycoses. 2007;50:317-20.

22. Butty P, Lebecq JC, Mallié M, et al. Evaluation of the susceptibility of dermatophytes to antifungal drugs: a new technique. $J$ Med Vet Mycol. 1995; 33 (6):403-9.

Costa23. Mock M, Monod M, Baudraz-Rosselet, et al. Tinea capitis dermatophytes: susceptibility to antifungal drugs tested in vitro and in vivo. Dermatology. 1998; 197 (4):361-7.

24. Badali H, Mohammadi R, Mashedi O, et al. In vitro susceptibility patterns of clinically important Trichophyton and Epidermophyton species against nine antifungal drugs. Mycoses. 2015; 58 (5):303-7.

25. Adimi P, Hashemi SJ, Mahmoudi M, et al. Invitro activity of 10 antifungal agents against 320 dermatophyte strains using microdilution method in Tehran. Iran J Pharm Res. 2013; 12 (3):537.

26. Yamada T, Maeda M, Alshahni MM, et al. Terbinafine resistance of Trichophyton clinical isolates caused by specific point mutations in the squalene epoxidase gene. Antimicrob Agents Chemother. 2017; 61(7):115-7.

27. Strobos MA and Oranje AP. Failure of terbinafine treatment in 2 children with tinea capitis. Ned Tijdschr Geneeskd. 1996; 140 (31):1611-2.

28. Kano R, Hsiao YH, Han HS, et al. Resistance mechanism in a terbinafine-resistant strain of Microsporum canis. Mycopathologia. 2018; 183 (3):623-7.

29. Osborne CS, Leitner I, Favre B, et al. Amino acid substitution in Trichophyton rubrum squalene epoxidase associated with resistance to terbinafine. Antimicrob Agents Chemother. 2005; 49 (7):2840-4.

30. Singh A, Masih A, Khurana A, et al. High terbinafine resistance in Trichophyton interdigitale isolates in Delhi, India harbouring mutations in the squalene epoxidase gene. Mycoses. 2018; 61 (7):477-84.

31. Taha M, Graser Y and Morsy S. Application of polymerase chain reaction for identification of dermatophytes isolated from human and animals in Egypt. Zag Vet J. 2009; 37:7-14. 